\title{
TRPV1 modulation of immune response in metastatic breast carcinoma: Enhanced inflammatory response may hinder therapeutic potentials of TRPV 1 agonists
}

Nuray Erin ( $\nabla$ nerin@akdeniz.edu.tr)

Akdeniz Universitesi Tip Fakultesi https://orcid.org/0000-0002-6116-1970

Muhlis Akman

Akdeniz Universitesi Tip Fakultesi

Seren Haksever

Akdeniz Universitesi Tip Fakultesi

\section{Research Article}

Keywords: Metastatic breast cancer, organ specific, microenvironment, TRPV1, AMG9810, Gambogic amide

Posted Date: July 21st, 2021

DOl: https://doi.org/10.21203/rs.3.rs-724375/v1

License: (c) (1) This work is licensed under a Creative Commons Attribution 4.0 International License.

Read Full License 


\section{Abstract}

Background and Purpose: The transient receptor potential vanilloid 1 (TRPV1) ion channels enhance cytotoxic immune response and may have therapeutic potential in cancer treatment. Hence, we here determined how activation of TRPV1 alters immune response of tumor-bearing mice.

Experimental Approach: Three different metastatic subset of 4T1 breast carcinoma cells were used to induce tumors in Balb-c mice. Mix leukocyte cultures (MLCs) using spleens and draining lymph nodes were prepared and stimulated with various challenges. Effects of four different TRPV1 agonists, antagonist (AMG9810) and Gambogic Amide (GA), a TrkA agonist that sensitizes TRPV1, on secreted levels of cytokines were determined.

Results: MLCs of tumor-bearing mice secreted markedly higher levels of IL- 6 and lower levels of IFN- $\gamma$ compared to control mice. We observed differential effects of TRPV1 agonists, antagonist and GA in control and mice bearing different subset of metastatic cells. TRPV1 and TrkA agonists increased IFN-- $Y$ and IL-17 secretion in control mice while they markedly increased IL- 6 secretion and suppressed IFN--y secretion in tumor-bearing mice. Unexpectedly, AMG9810 acted as an inverse agonist and did not antagonize the effects of TRPV1 agonists and GA did not sensitize TRPV1 channels.

Conclusions: Our results demonstrate constitutive activity of TRPV1 in immune cells, suggesting cross activation. Excessive chronic activation of TRPV1 in immune cells in the presence of metastatic breast carcinoma may have detrimental effects. Unexpected findings further document that a drug can have multiple intrinsic activities and depending on surrounding factors can act on the same receptor as an agonist, antagonist or inverse agonist.

\section{Bullet Point Summary}

- TRPV1 ion channels regulate inflammatory and cytotoxic immune responses and TrkA activity sensitize TRPV1

- Activation of TRPV1 ion channels has potential to inhibit tumor growth and metastasis.

- Effects of TRPV1 agonists on immune functions of mice bearing metastatic breast carcinoma were determined.

- Effects of TrkA activation alone and in-combination with TRPV1 agonists on immune functions were determined.

- TRPV1 in immune cells demonstrate constitutive activity suggesting extensive cross activation during metastatic disease

- Chronic activation of TRPV1 in immune cells during metastatic breast carcinoma may have detrimental effects.

\section{Introduction}


The transient receptor potential vanilloid subtype 1 (TRPV1) channels are belongs to Transient Receptor Potential (TRP) superfamily and were initially identified as the receptor for capsaicin, the pungent ingredient in chili peppers [1]. TRPV1 is involved in a spectrum of immune functions regulating of auto-, inflammatory and cytotoxic immune responses. TRPV1 expression is demonstrated in all types of mammalian immune cells, including lymphocytes, dendritic cells, macrophages and neutrophils $[2,3]$. TRPV1 found in T cells are involved in mitogen-induced T cell activation [2], and cytokine production [4].

TRPV1 activity decreases inflammation in several different models of inflammatory diseases $[5,6]$. Although chronic inflammation is one of the hallmarks of the carcinogenesis and metastasis [7], the role of TRPV1 activation on cancer immune response is largely unknown. We previously documented that inactivation of TRPV1 expressing sensory neurons increases metastasis of 4T1 breast carcinoma demonstrating that activating sensory neurons with TRPV1 agonists may inhibit tumor progression and metastasis [8]. In few studies, intra-tumoral injection of capsaicin enhanced local cancer immune response [3]. Hence, TRPV1 agonists might have therapeutic potential in cancer treatment and given the presence of TRPV1 channels in immune cells, immunological consequences of such treatments should be evaluated. The direct effects of capsaicin or other TRPV1 agonists on immune cells of tumor bearing mice were not determined before. The goal of this study was to determine the effects of TRPV1 agonists on immune response of tumor bearing mice using an in vitro model.

Activators or inhibitors of TRPV1 may have unpredicted or diverse consequences, which might be due to their off-target effects e.g modulation of other TRP channels [9]. Hence, we here examined the effects of four different agonists as well as AMG9810 (AMG), a potent and selective competitive TRPV1 antagonist [10].

Activation of TRPV1 channels with agonists may lead to desensitization [1]. Tyrosine receptor kinase A (TrkA) activation sensitize TRPV1 channels and increase TRPV1 expression in neuronal tissue [11]. TrkA is found in lymphocytes, monocytes and activated macrophages regulating immune functions [12]. Hence, TrkA agonists may enhance activity of TRPV1 channels in immune cells. To examine this possibility Gambogic Amide (GA) was used because it activates and enhances the expression TrkA and has potential to be used clinically in degenerative diseases [13] and cancer treatment [14].

Breast carcinoma is comprised of heterogeneous groups of cells with different metastatic potential. Differential features of $4 T 1$ breast carcinoma cells metastasized to heart (denoted as 4THM), liver (denoted as 4TLM) and brain (denoted as 4TBM) were reported before $[15,16]$. Hence, we here also examined how tumor immune response is altered in mice bearing different subset of metastatic cells in the presence and absence of TRPV1 activity.

\section{Materials And Methods}

\section{Mice}


Wild-type (WT) female BALB/c mice was purchased from the Kobay Laboratories Ankara/Turkey. All mice were used at 8-12 weeks of age. Study was approved by Akdeniz University, Animal Care Committee, and all animal experimentation was performed following the guidelines of an accredited animal care committee of Akdeniz University.

\section{Cell lines}

4T1 breast cancer cells were previously derived from a spontaneously formed breast tumor in a BALB/c female mouse [17]. 4THM cell line was derived from heart metastasis of 4T1 cells. 4TLM cell line was derived from liver metastases and 4TBM cell line was derived from brain metastasis of 4THM cells by Erin et al. [15]. 4TLM, 4THM and 4TBM cells were grown in DMEM-F12 supplemented with 5\% FBS (fetal bovine serum), $2 \mathrm{mM}$ l-glutamine, $1 \mathrm{mM}$ sodium pyruvate, $0.02 \mathrm{mM}$ nonessential amino acids and gentamicin $(80 \mu \mathrm{g} / \mathrm{ml})$.

Induction of tumor formation. 4TLM, 4TBM or 4THM cells prepared in hanks balanced salt solution ( $1 \times$ $10^{5}$ cells in $0,1 \mathrm{ml}$ per mouse) were injected orthotopically into the right upper mammary gland of $8-10$ weeks old female Balb-c mice under ketamine-xylazine anesthesia. For each group 5 mice were used. Palpable tumors were formed six days after injection of cancer cells.

\section{Leukocyte cultures}

Mix leukocyte culture (MLC) was prepared 18 days after injection of metastatic breast carcinoma cells. This time point was chosen based on our previous results and reflects the time when systemic metastases are detectable microscopically and mice are not markedly immune suppressed [18-20]. Specifically spleen and draining lymph nodes of animals injected with metastatic cancer cells as well as control mice (vehicle injected) were removed aseptically and single-cell suspensions were prepared MLCs as described before $[19,20]$. Specifically lymph nodes and spleens were passed through custom-made steel cell strainer, washed two times with PBS, and counted. $\left(4 \times 10^{6} /\right.$ well in 48 -well tissue culture plate) were cultured alone (control for basal cytokine release) or stimulated with lipopolysaccharide (LPS, 3 $\mu \mathrm{g} / \mathrm{ml})$, Concanavalin A (ConA, $5 \mu \mathrm{g} / \mathrm{ml})$, or irradiated ( 20 Gray) cancer cells $\left(5 \times 10^{4} /\right.$ well). RPMl medium supplemented with gentamycin $(80 \mu \mathrm{g} / \mathrm{ml}), 10 \%$ FBS and 2-mercaptoethanol $(2 \mu \mathrm{l}$ to $500 \mathrm{ml}$ RPMI) and was used for culturing immune cells.

\section{TRPV1 agonists, antagonist and Gambogic amide treatments}

MLCs challenged with LPS or ConA were treated with Capsaicin (Cap) $(1 \mu \mathrm{M}$ and $10 \mu \mathrm{M})$, resinferatoxin

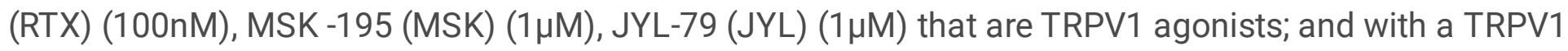
antagonist AMG9810 (AMG) (10 $\mu$ M). MLCs were also treated with Gambogic Amide (GA) (150nM). Unchallenged MLCs were treated with TRPV1 agonists, AMG and GA. AMG was obtained from Cayman (Cat No: 14715), caspaicin was from Sigma (Cat No: M2028), RSX, MSK and GA was obtained from Santa Cruz (Cat No: 24015, 202712A and 221655A respectively). Each treatment repeated 6 times. 


\section{Measurement of cytokines}

Specifically changes in TNF-a, IFN- $y$, IL-6, IL-17 and IL-10 levels were determined in supernatants using ELISA. TNF-a levels were measured $18 \mathrm{~h}$ after challenge, while IFN- $y$, IL-6, IL-17 and IL-10 levels were measured in supernatants obtained $40 \mathrm{~h}$ after challenge. Standard ELISA kits (Biolegend, San Diego, CA, USA) were used for cytokine measurements. All other standard reagents as well as LPS and ConA were obtained from Sigma.

\section{Statistics}

ANOVA with Dunnett's post test was used for the analysis of cytokine results. $P$ values $<0.05$ were considered biologically significant. Statistical analyses were performed using GraphPad InStat 3 software.

\section{Results}

The effects of TRPV1 activation on cytokine response of mix leukocyte culture obtained from control mice.

MLCs were prepared from lymph nodes and spleens of tumor-free (control) mice and challenged by ConA and LPS. Capsaicin $(1 \mu \mathrm{M}), \mathrm{RSX}(100 \mathrm{nM}), \mathrm{MSK}(1 \mu \mathrm{M})$ markedly enhanced IFN- $\gamma$ and IL-17 response to ConA while they induced a modest but significant increase in TNF-a secretion (Fig. 1A-C). The concentrations of the drugs were chosen based on previous data [21,22]. Similarly, Capsaicin, RSX and MSK also markedly increased LPS-induced IL-10 secretion (Fig. 1D). JYL, another potent agonist of TRPV1, on the other hand did not alter the levels of IL-17, IL-10 and TNF-a. Surprisingly JYL decreased the IFN- $y$ secretion demonstrating differential effects of agonists (Fig. 1A-D). TRPV1 agonists except capsaicin did not alter the secretion of LPS-induced IL-6 release (Fig. 1E).

TRPV1 antagonist AMG $(10 \mu \mathrm{M})$ [23], did not affect IFN- $y$ and TNF- $\alpha$ secretion, increased IL-17 release and decreased IL-10 and IL- 6 secretion. On the other hand, AMG antagonized the effects of TRPV1 agonists (Capsaicin, RSX, MSK) on IFN- $y$, IL-17, IL-10 and TNF-a release. Surprisingly, AMG did not only antagonized the increases in these cytokines but also induced further decreases in IFN- $\gamma$ and TNF-a secretion when combined with agonists, demonstrating AMG is not only act as an competitive inhibitor of TRPV1 activation but also acts as an inverse agonist in the presence of activating ligands [24].

GA (150nM), the TrkA agonist, also markedly increased IFN- - , IL-17 and TNF-a levels similar to TRPV1 agonists. Combination of GA with capsaicin or MSK did not further alter the secretion of these cytokines. GA is likely to increase activity of T cells since its' effects on LPS-induced cytokine secretion was mostly minimal except LPS-induced IL-10 secretion which was significantly decreased (Fig. 1D and Supp Fig. 1).

Supp. Figure 1 demonstrates LPS-induced secretions of IFN- $y$ and TNF- $a$, which were markedly lower than ConA-induced secretions. Similarly ConA-induced IL- 6 secretion was markedly lower compared to LPS- 
induced secretion. ConA caused higher levels of IL-10 secretion compared to LPS in control mice (Supp. Figure 1); the effects of TRPV1 agonists and antagonist however were similar under both challenges.

\section{Modulation of TNF-a secretion of MLCs prepared from mice bearing different subset of metastatic breast carcinoma cells.}

Based on our findings obtained in control mice (not injected with cancer cells), TRPV1 agonists that consistently altered the cytokine levels were chosen in the following sets of experiments. Specifically, two different concentrations of capsaicin $(1,10 \mu \mathrm{M}), \mathrm{RTX}(100 \mathrm{nM})$, MSK $(1 \mu \mathrm{M})$, as well as TRPV1 antagonist AMG $(10 \mu \mathrm{M})$ and TrkA agonist GA (150nM) were used. In addition to LPS and ConA, MLCs were also challenged with corresponding cancer cells, which were irradiated. The effects of TRPV1 modulators on ConA-induced TNF-a secretion of MLCs varied depending on the subset of metastatic cells injected. Specifically, TRPV1 agonists markedly decreased TNF-a secretion in MLCs of mice bearing 4TBM cells, did not altered the levels (except high concentration of capsaicin) in MLCs of mice bearing 4THM cells and markedly enhanced the TNF-a secretion in in MLCs of mice bearing 4TLM cells (Fig. 2A-C).

Interestingly, TRPV1 antagonist AMG decreased TNF-a secretion in all groups, the effect was much more prominent in MLCs of 4TBM and 4TLM injected mice demonstrating inverse agonist properties of AMG on immune cells of carcinoma bearing mice. When immune cells were co-treated with TRPV1 agonist and AMG, the results were similar to the results obtained in AMG-only treated group. These results were different from the results obtained in control mice and indicate that outcome of TRPV1 inhibition by AMG depends on how the carcinoma modulates the immune response.

Differential effects of GA were also observed such that GA markedly decreased TNF-a level only in 4TLM cells and this effect was partly antagonized by capsaicin, which alone enhanced TNF-a secretion. It was striking to observe the differential effects of GA and AMG depending on the challenge. GA and AMG more effectively suppressed TNF-a response to ConA-stimulation of T cells compared to non-specific LPSstimulation of lenfoid and monocyte-originated cells as seen in 4TLM and 4TBM-injected groups (Supp. Figure 2). Furthermore, GA actually enhanced TNF-a secretion in LPS-challenged MLCs of 4TLM and 4THM-injected groups. These results demonstrate that GA may enhance the inflammatory response in the presence of TLR-4 stimulation [25].

\section{Modulation of IFN-y secretion of MLCs prepared from mice bearing different subset of metastatic breast carcinoma cells.}

TRPV1 agonists decreased IFN- $\gamma$ secretion in MLCs of 4TBM and 4TLM-injected mice and did not alter the levels in 4THM-injected mice, which secreted the least amount of IFN- $\gamma$. Interestingly after treatment with TRPV1 agonists IFN- $\gamma$ levels were similar (around $80-120 \mathrm{pg} / \mathrm{ml}$ in average of three agonists) in all three groups demonstrating that TRPV1 activity may moderate the signals for IFN- $y$ secretion (Fig. 3A-C). AMG, TRPV1 antagonist, also markedly suppressed IFN- $\gamma$ response in all groups, which was not altered by TRPV1 agonists (Fig. 3A-C). 
The effects of GA on IFN-y secretion were similar to TRPV1 agonists in 4TBM and 4TLM groups. On the other hand, combination of GA with high concentration of capsaicin (10 $\mu \mathrm{M})$ prevented the GA-induced decreases in IFN-y secretion. Furthermore capsaicin $(10 \mu \mathrm{M})$ and GA co-treatment markedly enhances IFN-y response compared to group treated with vehicle in MLCs of 4TBM-injected mice. GA enhanced IFN$\mathrm{Y}$ response in MLCs of 4THM-injected-mice (Fig. 3A-C).

\section{Modulation of IL-6 secretion of MLCs prepared from mice bearing different subset of metastatic breast carcinoma cells.}

Challenge with irradiated tumor cells also induced IL-6 secretion, which was markedly higher than levels obtained following LPS challenge in 4TBM and 4THM cells. Irradiated tumor cells do not secrete appreciable levels of IL-6 as reported before [19]. TRPV1 agonists markedly potentiated IL-6 secretion in response to LPS and irradiated tumor cells in MLCs of mice injected with 4THM and 4TLM cells but not 4TBM cells (Fig. 4A-F).

AMG inhibited IL-6 secretion in response to irradiated cancer cells. The effects of AMG and TRPV1 agonist combination were similar to the effects of AMG under most conditions (Fig. 4A-C).

AMG decreased LPS-induced IL-6 secretion up to around 1500-2000 pg/ml in MLCs of 4TBM or 4TLM injected mice. LPS induced IL-6 secretion in MLCs of 4THM-injected mice was around $1500 \mathrm{pg} / \mathrm{ml}$ and AMG did not altered the levels in this group suggesting that under LPS challenge, concentration of IL-6 may determine the outcome of AMG treatment (Fig. 4D-F).

GA, similar to TRPV1 agonists, significantly increased the LPS-induced IL-6 secretion in MLCs of 4THM and 4TBM-injected mice but the extent of increase was significantly less than the levels obtained following treatment with TRPV1 agonists. Similarly, there was a slight but significant increase following treatment with GA in MLCs challenged with irradiated 4THM and 4TLM cells (Fig. 4D-F).

Con-A lead to a comparable IL-6 response in all groups and the effects of treatments were similar to the effects observed following LPS challenge. Comparisons of the results for AMG and GA are given in Supp. Figure 3.

\section{Modulation of IL-17 secretion of MLCs prepared from mice bearing different subset of metastatic breast carcinoma cells.}

Con-A challenge induced high levels of IL-17 secretion and the levels obtain were between 800-1000 $\mathrm{pg} / \mathrm{ml}$. TRPV1 agonists did not alter while AMG markedly suppressed IL-17 secretion. The degree of suppressive effects of AMG however differed amongst the groups and were most prominent (more than $70 \%$ decrease) in 4TBM or 4THM injected groups. Suppressive effects of AMG were less prominent in MLCs of mice bearing 4TLM tumors demonstrating partial resistance of this group to AMG treatment. These results further document the inverse agonist propertied of AMG, which could not be reversed with capsaicin or MSK. GA did not affect IL-17 secretion in response to ConA challenge (Fig. 4A-C). 
Although to a much lesser degree, irradiated tumor cells also evoked IL-17 secretion, and TRPV1 agonists prevented this increase in groups injected with 4THM and 4TLM cells. AMG markedly suppressed IL-17 secretion in response to irradiated cancer cells. GA induced slight but significant decreases in IL-17 secretion from MLCs of mice bearing 4TLM tumors in response to irradiated cancer cells and this effect was prevented in the presence of capsaicin demonstrating counteracting effects of TRPV1 and TrkA receptor activities under these conditions.

We did not detected major changes in LPS-induced secretion of IL-10, main immunosuppressive/antiinflammatory cytokine, following treatment with TRPV1 agonists, AMG and GA in this experimental setting. Representative results were shown in Supp. Figure 4.

\section{Differences in cytokine response of MLC among the control mice and mice bearing different subset of metastatic breast carcinoma cells.}

Release of inflammatory cytokines IL-6 and TNF- $a$ in response to LPS stimulation was markedly higher (20-30 times and 3-4 times respectively) and IFN- $\gamma$ secretion was markedly lower in mice bearing metastatic breast carcinoma (Supp. Figure 5). TNF-a secretion in response to ConA was somewhat similar among the groups. IL-17 secretion was also higher in tumor-bearing mice while IL-10 secretion varied depending on the subtype of metastatic breast cancer cells. Similarly, we also detected subtypedependent changes in cytokine secretion. Specifically, ConA-induced IFN-y response and LPS-induced IL-6 response was highest in animals injected with 4TBM cells while IL-10 and IFN- $\gamma$ response to ConA was lowest in 4THM-injected mice. These results further document differential systemic effects of different metastatic sub clones of $4 \mathrm{~T} 1$ breast carcinoma through immune response and may explain differences in their metastatic potentials and treatment response as reported before $[8,16,26,27]$.

\section{Discussion}

We here examined the effects of TRPV1 modulators (agonists and antagonist) as well as TrkA agonist on immune activity in the presence and absence of metastatic breast carcinoma. The effects of four different TRPV1 agonists were tested given possible differences in their activity and except JYL, all demonstrate somewhat similar changes, hence we here discussed the average effects of three agonists.

MLC of tumor-bearing mice secreted markedly higher levels IL- 6 and lower levels of IFN- $\gamma$ compared to control mice. Chronic increases in IL-6 levels play a critical role in progression and metastasis of carcinomas and IFN- $\gamma$ is critical for cytotoxic immune response. Hence, increased IL-6 in conjunction with decreased IFN- $\gamma$ secretion markedly impairs anti-tumoral immunity enhancing metastatic ability $[28,29]$. These results are in accordance with the increased inflammatory response and decreased cytotoxic immunity in aggressive carcinomas, further validating our model as grade four metastatic breast carcinoma $[16,17,19,20,30,31]$. Levels of TNF-a, another tumor-promoting cytokine, were markedly increased in response to LPS but not ConA in tumor bearing mice demonstrating the predominant role of myeloid derived cells in agreement with our previous results [18]. 
TRPV1 agonists and TrkA agonist have distinct effects in the presence of metastatic cancer compared to healthy non-tumor bearing mouse. TRPV1 agonists markedly increased IFN- $\gamma$ response in control mice while the opposite occurred in tumor-bearing mice though some cell-type dependent changes were present. TRPV1 agonists markedly increased IL-17 secretion in control mice while they did not alter the levels of IL-17 secretion in tumor bearing mice. On the other hand, TRPV1 agonists markedly increased IL6 secretion in tumor-bearing mice but not in control mice. These results demonstrate that activation of TRPV1 channels in the absence of aggressive inflammatory carcinoma may enhance immune response against newly formed precancerous lesions by increasing IFN-ץ [32] and acute inflammatory response e.g. by increasing IL-17 secretion [33]. IL-17 is also by poly-functional T cells in the presence of cancer and IL-17 mediates cytotoxic immunity against cancer cells together with IFN- $\gamma$ [34]. Similar effects of TrkA activation with GA was also observed in control mice. Hence, TRPV1 agonists such as capsaicin as well as GA may prevent or decrease the risk of cancer formation. On the other hand, chronic use of high doses of TRPV1 agonists may enhance chronic inflammation by increasing IL-6 in carcinomas characterized by systemic inflammation and may actually enhance metastasis. Hence, a screening method should be used to determine how the TRPV1 activity alters systemic immune response in a patient-specific approach e.g using patients' peripheral blood mononuclear cell. This approach also seems to be valid for GA use in cancer treatment since it also enhanced IL- 6 secretion in mice bearing certain subset of metastatic breast carcinoma.

It was shown that systemic inflammatory response and consequent organ damage increases in TRPV1 knockout mice $[35,36]$. Oral pretreatment with capsaicin reduces sciatic nerve inflammation by decreasing the number of infiltrating $T$ cells, macrophages and production of proinflammatory cytokines in experimental autoimmune neuritis [37]. These findings are in contrast with what we observed in this study. This is likely to be due to differential consequences of activation of systemic versus local TRPV1 e.g on immune cells. Furthermore, TRPV1 ion channels are polymodal receptors perceiving and integrating different stimuli within microenvironment. More importantly, levels of TRPV1 expression vary depending on the cell type. Specifically TRPV1 is highly expressed in sensory neurons compared to immune and other non-neuronal cells [1]. Nano molar concentration of capsaicin and related TRPV1 agonists are sufficient to activate nerve fibers [38] while much higher concentrations are required for TRPV1 activation of immune cells [35]. We previously documented that Substance P, the main neuropeptide released from TRPV1 + sensory neurons, enhances anti-tumoral immunity in conjunction with radiotherapy leading complete remission in $50 \%$ of mice bearing metastatic breast carcinoma [20]. Activation of neuronal TRPV1 also leads to secretion of neuropeptides with anti-inflammatory properties such as somatostatin [39]. TRPV1 expressing sensory neurons directly alter functions of immune cells by releasing neuropeptides upon activation with TRPV1 agonists [40, 41]. For example, CGRP released from sensory nerve endings induces production of IL-17A by $\gamma \delta$ T cells that elicits host defense against pathogens [42]. Hence anti-inflammatory and anti-tumoral/anti-bacterial/cytotoxic effects reported following systemic treatment with TRPV1 agonists might largely be due to activation of neuronal TRPV1 channels. Our findings demonstrate that activation TRPV1 channels of immune cells may create excessive inflammatory conditions under certain pathological conditions e.g. in the presence of 
inflammatory metastatic carcinoma. Because expression of TRPV1 channels are markedly higher in neuronal cells making them sensitive to low doses of TRPV1 agonist, using lower doses of TRPV1 agonists for treatment of malignancies and inflammatory disease may overcome possible unwanted effects of chronic TRPV1 activation of immune cells.

Classically, antagonists should not have an effect by themselves but should prevent the effects of agonists [24]. In accordance, AMG was reported to suppress the production of cytokines induced by activation of TRPV1 channels by LPS [10]. We however did not observed these classical changes; TRPV1 antagonist AMG did not affect IFN- $y$ and TNF-a secretion, increased IL-17 release and suppressed IL-6 and IL-10 release in MLC of control mice. In-tumor bearing mice suppressive effects of AMG was much more prominent especially when combined with a TRPV1 agonist. These results demonstrate the presence of complex interactions between TRPV1 and other signaling systems and indicate cross activation of TRPV1 channels under various challenges. Specifically, our results suggest that activation of immune cells with LPS, ConA or irradiated tumor cells strongly cross activates TRPV1 channels directly or indirectly and inhibition of TRPV1 channels with AMG alters cytokine response. This is similar to constitutive receptor activity demonstrated in various systems in which receptors can be active without an activating ligand [24]. Inverse agonists block constitutive activity hence AMG seems to act as an inverse agonist. These results further provide evidence for emerging concept stating that a drug, acting at a single receptor subtype, can have multiple intrinsic activity and can be simultaneously an agonist, an antagonist, and an inverse agonist [24]. Our results also further document that these features of receptordrug interactions need to be considered when drugs are used in treatment of immunological diseases and cancer or as research tools.

Inflammation increases TRPV1 expression in neurons enhancing their activity [43]. Similarly, proinflammatory chemokine, CCL3 sensitizes TRPV1 [44] and TNF-a increases cell surface expression of TRPV1 in ganglion neurons [45]. Effects of inflammatory mediators on TRPV1 of immune cells are not known. LPS activates TRPV1 and other TRP channels such as TRPA1 in immune cells [10]. Our results suggest that cytokine/chemokine milieu of immune cells preconditioned with aggressive breast carcinoma and challenged with a stimulant strongly cross activates TRPV1 channels which might be responsible from increased secretion of inflammatory cytokines. Because AMG suppressed secretion of all the cytokines studied except IL-10, it is therapeutic potential seems to be low in prevention of carcinoma-induce chronic inflammatory response because of risk of general immune suppression. Furthermore, inhibition of neuronal TRPV1 activity with AMG may also enhance tumor growth and metastasis [30]. These possibilities should be further evaluated.

Extensive cross activation of TRPV1 channels of activated immune cells of mice bearing cancer cells may also explain unexpected effects of GA, a TrkA agonist known to sensitize TRPV1 channels [11]. Specifically, in 4TLM and 4TBM-injected mice, GA markedly decreased TNF-a levels and this effect was antagonized by capsaicin demonstrating opposing effects of TRPV1 and TrkA pathways in this model. Similar antagonistic interactions were observed in IL-6 response. Furthermore, GA did not enhance the effects of TRPV1 agonists demonstrating inability of TrkA to sensitize TRPV1 channels in immune cells. 
It was striking to observe the differential effects of GA and AMG depending on the challenge. GA and AMG more effectively suppressed TNF response to ConA-stimulation of T cells within MLCs compared to non-specific LPS-stimulation of lenfoid and monocyte-originated cells. Furthermore, GA actually enhanced TNF-a secretion in LPS-challenged MLCs of 4TLM and 4THM-injected groups. These results demonstrate that GA may enhance the inflammatory response in the presence of TLR-4 stimulation of tumor bearing mice.

In conclusion, our results documented that outcome of treatment with TRPV1 agonists and antagonists on immune response vary depending on the status of carcinoma-induced immunological changes and could not be easily predicted. Our results further validated our model as highly aggressive grade four metastatic breast carcinoma and in this model; chronic use of high doses of TRPV1 agonists systemically may have detrimental effects because of increased inflammatory response. Furthermore, in the presence of heterogeneous group of immune cells as in MLCs, cross activation of TRPV1 occurs leading constitutive activity, which explains inverse agonist properties of AMG and other unexpected results obtained in combination treatments.

\section{Declarations}

Funding (information that explains whether and by whom the research was supported): This study supported by fund from The Scientific and Technological Research Council of Turkey (TÜBITAK), Project No: $115 S 943$

Conflicts of interest/Competing interests (include appropriate disclosures): Authors declare no conflict of interest

Availability of data and material (data transparency: Data sharing is not applicable to this article because all the data generated in this study were included in the paper. No additional new data were created or analysed in this study.

Code availability (software application or custom code):Not applicable

Authors' contributions: NE planned and designed the experiments, analyzed the results and wrote the manuscript. MA and SH performed the experiments.

Ethics approval (include appropriate approvals or waivers): Study was approved by Akdeniz University, Animal Care Committee, and all animal experimentation was performed following the guidelines of an accredited animal care committee of Akdeniz University.

Consent to participate (include appropriate statements): Not applicable

Consent for publication (include appropriate statements): Not applicable 
Acknowledgements: This study supported by fund from The Scientific and Technological Research Council of Turkey (TÜBITAK), Project No: 115 S943.

\section{References}

1. Caterina, M. J., M. A. Schumacher, M. Tominaga, T. A. Rosen, J. D. Levine, and D. Julius. 1997. The capsaicin receptor: a heat-activated ion channel in the pain pathway. Nature 389 (6653): 816-824.

2. Bertin, S., Y. Aoki-Nonaka, P. R. de Jong, L. L. Nohara, H. Xu, S. R. Stanwood, S. Srikanth, J. Lee, K. To, and L. Abramson, et al. 2014. The ion channel TRPV1 regulates the activation and proinflammatory properties of CD4(+) T cells. Nat Immunol 15 (11): 1055-1063.

3. Ghosh, A. K., and S. Basu. 2012. Tumor macrophages as a target for Capsaicin mediated immunotherapy. Cancer Lett 324 (1): 91-97.

4. Majhi, R. K., S. S. Sahoo, M. Yadav, B. M. Pratheek, S. Chattopadhyay, and C. Goswami. 2015. Functional expression of TRPV channels in T cells and their implications in immune regulation. FEBS $J 282$ (14): 2661-2681.

5. Fernandes, E. S., L. Liang, S. J. Smillie, F. Kaiser, R. Purcell, D. W. Rivett, S. Alam, S. Howat, H. Collins, and S. J. Thompson, et al. 2012. TRPV1 deletion enhances local inflammation and accelerates the onset of systemic inflammatory response syndrome. J Immuno/ 188 (11): 5741-5751.

6. Clark, N., J. Keeble, E. S. Fernandes, A. Starr, L. Liang, D. Sugden, P. de Winter, and S. D. Brain. 2007. The transient receptor potential vanilloid 1 (TRPV1) receptor protects against the onset of sepsis after endotoxin. FASEB J 21 (13): 3747-3755.

7. Hanahan, D., and R. A. Weinberg. 2011. Hallmarks of cancer: the next generation. Cell 144 (5): 646674.

8. Erin, N., W. Zhao, J. Bylander, G. Chase, and G. Clawson. 2006. Capsaicin-induced inactivation of sensory neurons promotes a more aggressive gene expression phenotype in breast cancer cells. Breast Cancer Res Treat 99 (3): 351-364.

9. Boonen, B., Y. A. Alpizar, A. Sanchez, A. Lopez-Requena, T. Voets, and K. Talavera. 2018. Differential effects of lipopolysaccharide on mouse sensory TRP channels. Cell Calcium 73: 72-81.

10. Ninomiya, Y., S. I. Tanuma, and M. Tsukimoto. 2017. Differences in the effects of four TRPV1 channel antagonists on lipopolysaccharide-induced cytokine production and COX-2 expression in murine macrophages. Biochem Biophys Res Commun 484 (3): 668-674.

11. Winston, J., H. Toma, M. Shenoy, and P. J. Pasricha. 2001. Nerve growth factor regulates VR-1 mRNA levels in cultures of adult dorsal root ganglion neurons. Pain 89 (2-3): 181-186.

12. Meeker, R., and K. Williams. 2014. Dynamic nature of the p75 neurotrophin receptor in response to injury and disease. J Neuroimmune Pharmaco/ 9 (5): 615-628.

13. Jang, S. W., M. Okada, I. Sayeed, G. Xiao, D. Stein, P. Jin, and K. Ye. 2007. Gambogic amide, a selective agonist for TrkA receptor that possesses robust neurotrophic activity, prevents neuronal cell death. Proc Natl Acad Sci U S A 104 (41): 16329-16334. 
14. Jarboe, J. S., J. J. Jaboin, J. C. Anderson, S. Nowsheen, J. A. Stanley, F. Naji, R. Ruijtenbeek, T. Tu, D. E. Hallahan, and E. S. Yang, et al. 2012. Kinomic profiling approach identifies Trk as a novel radiation modulator. Radiother Oncol 103 (3): 380-387.

15. Erin, N., S. Kale, G. Tanriover, S. Koksoy, O. Duymus, and A. F. Korcum. 2013. Differential characteristics of heart, liver, and brain metastatic subsets of murine breast carcinoma. Breast Cancer Res Treat 139 (3): 677-689.

16. Erin, N., N. Wang, P. Xin, V. Bui, J. Weisz, G. A. Barkan, W. Zhao, D. Shearer, and G. A. Clawson. 2009. Altered gene expression in breast cancer liver metastases. Int J Cancer 124 (7): 1503-1516.

17. Aslakson, C. J., and F. R. Miller. 1992. Selective events in the metastatic process defined by analysis of the sequential dissemination of subpopulations of a mouse mammary tumor. Cancer Res 52 (6): 1399-1405.

18. Erin, N., S. Dilmac, A. Curry, O. Duymus, G. Tanriover, A. Prodeus, J. Gariepy, and R. M. Gorczynski. 2020. CD200 mimetic aptamer PEG-M49 markedly increases the therapeutic effects of pegylated liposomal doxorubicin in a mouse model of metastatic breast carcinoma: an effect independent of CD200 receptor 1. Cancer Immunol Immunother 69 (1): 103-114.

19. Erin, N., A. Podnos, G. Tanriover, O. Duymus, E. Cote, I. Khatri, and R. M. Gorczynski. 2015. Bidirectional effect of CD200 on breast cancer development and metastasis, with ultimate outcome determined by tumor aggressiveness and a cancer-induced inflammatory response. Oncogene 34 (29): 3860-3870.

20. Erin, N., A. F. Korcum, G. Tanriover, S. Kale, N. Demir, and S. Koksoy. 2015. Activation of neuroimmune pathways increases therapeutic effects of radiotherapy on poorly differentiated breast carcinoma. Brain Behav Immun 48: 174-185.

21. Lee, J., J. Lee, J. Kim, S. Y. Kim, M. W. Chun, H. Cho, S. W. Hwang, U. Oh, Y. H. Park, and V. E. Marquez, et al. 2001. N-(3-Acyloxy-2-benzylpropyl)-N'-(4-hydroxy-3-methoxybenzyl) thiourea derivatives as potent vanilloid receptor agonists and analgesics. Bioorg Med Chem 9 (1): 19-32.

22. Lee, J., J. Lee, M. S. Kang, K. P. Kim, S. J. Chung, P. M. Blumberg, J. B. Yi, and Y. H. Park. 2002. Phenolic modification as an approach to improve the pharmacology of the 3-acyloxy-2-benzylpropyl homovanillic amides and thioureas, a promising class of vanilloid receptor agonists and analgesics. Bioorg Med Chem 10 (4): 1171-1179.

23. Henrich, M., and K. J. Buckler. 2009. Acid-evoked Ca2 + signalling in rat sensory neurones: effects of anoxia and aglycaemia. Pflugers Arch 459 (1): 159-181.

24. Berg, K. A., and W. P. Clarke. 2018. Making Sense of Pharmacology: Inverse Agonism and Functional Selectivity. Int J Neuropsychopharmacol 21 (10): 962-977.

25. Zamyatina, A., and H. Heine. 2020. Lipopolysaccharide Recognition in the Crossroads of TLR4 and Caspase-4/11 Mediated Inflammatory Pathways. Front Immunol 11: 585146.

26. Erin, N., O. Duymus, S. Ozturk, and N. Demir. 2012. Activation of vagus nerve by semapimod alters substance P levels and decreases breast cancer metastasis. Regul Pept 179 (1-3): 101-108. 
27. Nizam, E., S. Koksoy, and N. Erin. 2020. NK1R antagonist decreases inflammation and metastasis of breast carcinoma cells metastasized to liver but not to brain; phenotype-dependent therapeutic and toxic consequences. Cancer Immunol Immunother 69 (8): 1639-1650.

28. Erin, N., J. Grahovac, A. Brozovic, and T. Efferth. 2020. Tumor microenvironment and epithelial mesenchymal transition as targets to overcome tumor multidrug resistance. Drug Resist Updat 53: 100715.

29. Weber, R., C. Groth, S. Lasser, I. Arkhypov, V. Petrova, P. Altevogt, J. Utikal, and V. Umansky. 2021. IL-6 as a major regulator of MDSC activity and possible target for cancer immunotherapy. Cell Immunol 359: 104254.

30. Erin, N. 2020. Role of sensory neurons, neuroimmune pathways, and transient receptor potential vanilloid 1 (TRPV1) channels in a murine model of breast cancer metastasis. Cancer Immunol Immunother 69 (2): 307-314.

31. Erin, N., E. Tavsan, O. Akdeniz, V. M. S. Isca, and P. Rijo. 2021. Rebound increases in chemokines by CXCR2 antagonist in breast cancer can be prevented by PKCdelta and PKCepsilon activators. Cytokine 142: 155498.

32. Koebel, C. M., W. Vermi, J. B. Swann, N. Zerafa, S. J. Rodig, L. J. Old, M. J. Smyth, and R. D. Schreiber. 2007. Adaptive immunity maintains occult cancer in an equilibrium state. Nature 450 (7171): 903907.

33. Zou, W., and N. P. Restifo. 2010. T(H)17 cells in tumour immunity and immunotherapy. Nat Rev Immunol 10 (4): 248-256.

34. Marshall, N. A., K. C. Galvin, A. M. Corcoran, L. Boon, R. Higgs, and K. H. Mills. 2012. Immunotherapy with PI3K inhibitor and Toll-like receptor agonist induces IFN-gamma + IL-17 + polyfunctional T cells that mediate rejection of murine tumors. Cancer Res 72 (3): 581-591.

35. Bujak, J. K., D. Kosmala, I. M. Szopa, K. Majchrzak, and P. Bednarczyk. 2019. Inflammation, Cancer and Immunity-Implication of TRPV1 Channel. Front Oncol 9: 1087.

36. Wang, Y., and D. H. Wang. 2013. TRPV1 ablation aggravates inflammatory responses and organ damage during endotoxic shock. Clin Vaccine Immunol 20 (7): 1008-1015.

37. Motte, J., B. Ambrosius, T. Gruter, H. Bachir, M. Sgodzai, X. Pedreiturria, K. Pitarokoili, and R. Gold. 2018. Capsaicin-enriched diet ameliorates autoimmune neuritis in rats. J Neuroinflammation 15 (1): 122.

38. McDonald, H. A., T. R. Neelands, M. Kort, P. Han, M. H. Vos, C. R. Faltynek, R. B. Moreland, and P. S. Puttfarcken. 2008. Characterization of A-425619 at native TRPV1 receptors: a comparison between dorsal root ganglia and trigeminal ganglia. Eur J Pharmacol 596 (1-3): 62-69.

39. Szolcsanyi, J., E. Pinter, Z. Helyes, and G. Petho. 2011. Inhibition of the function of TRPV1-expressing nociceptive sensory neurons by somatostatin 4 receptor agonism: mechanism and therapeutical implications. Curr Top Med Chem 11 (17): 2253-2263.

40. Cohen, J. A., T. N. Edwards, A. W. Liu, T. Hirai, M. R. Jones, J. Wu, Y. Li, S. Zhang, J. Ho, and B. M. Davis, et al. 2019. Cutaneous TRPV1(+) Neurons Trigger Protective Innate Type 17 Anticipatory 
Immunity. Cell 178 (4): 919-932 e914.

41. Verheijden, S., and G. E. Boeckxstaens. 2018. Neuroimmune interaction and the regulation of intestinal immune homeostasis. Am J Physiol Gastrointest Liver Physiol 314 (1): G75-G80.

42. Dantzer, R. 2018. Neuroimmune Interactions: From the Brain to the Immune System and Vice Versa. Physiol Rev 98 (1): 477-504.

43. Funahashi, Y., R. Takahashi, S. Mizoguchi, T. Suzuki, E. Takaoka, J. Ni, Z. Wang, D. B. DeFranco, W. C. de Groat, and P. Tyagi, et al. 2019. Bladder overactivity and afferent hyperexcitability induced by prostate-to-bladder cross-sensitization in rats with prostatic inflammation. J Physio/ 597 (7): 20632078.

44. Zhang, N., S. Inan, A. Cowan, R. Sun, J. M. Wang, T. J. Rogers, M. Caterina, and J. J. Oppenheim. 2005. A proinflammatory chemokine, CCL3, sensitizes the heat- and capsaicin-gated ion channel TRPV1. Proc Natl Acad Sci U S A 102 (12): 4536-4541.

45. Meng, J., J. Wang, M. Steinhoff, and J. O. Dolly. 2016. TNFalpha induces co-trafficking of TRPV1/TRPA1 in VAMP1-containing vesicles to the plasmalemma via Munc18-1/syntaxin1/SNAP25 mediated fusion. Sci Rep 6: 21226.

\section{Figures}

A
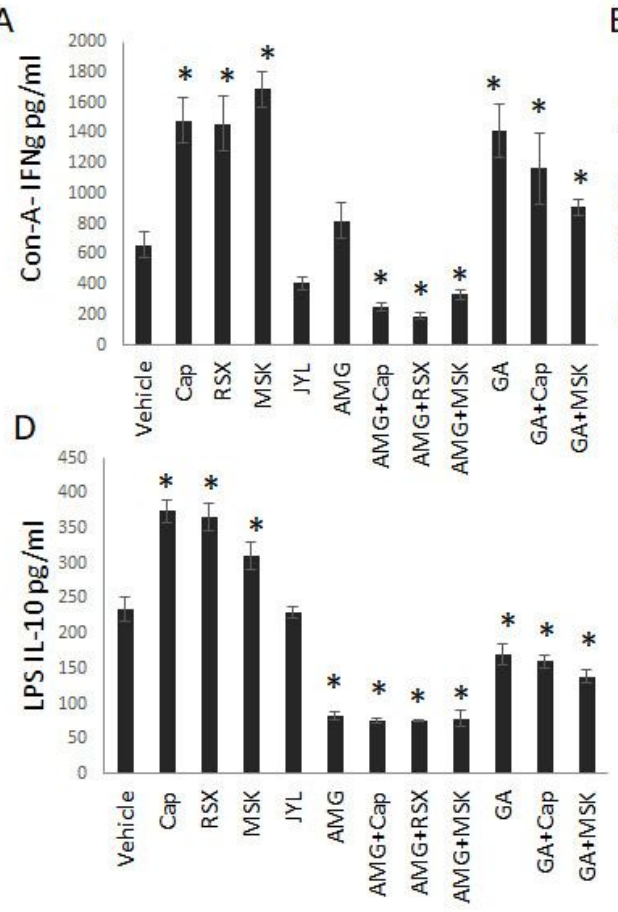

B

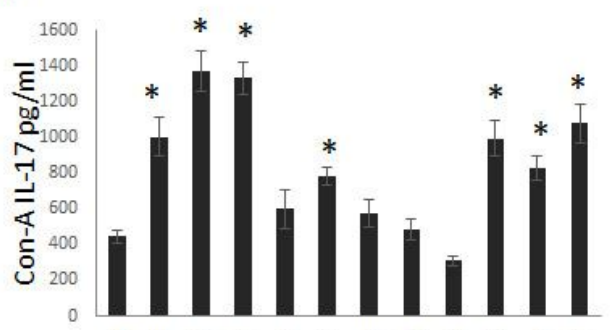

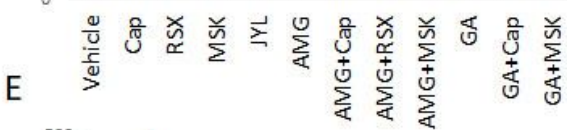

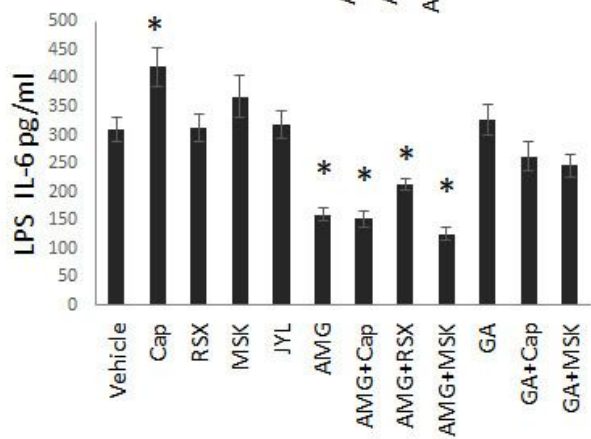

C ${ }_{45}$

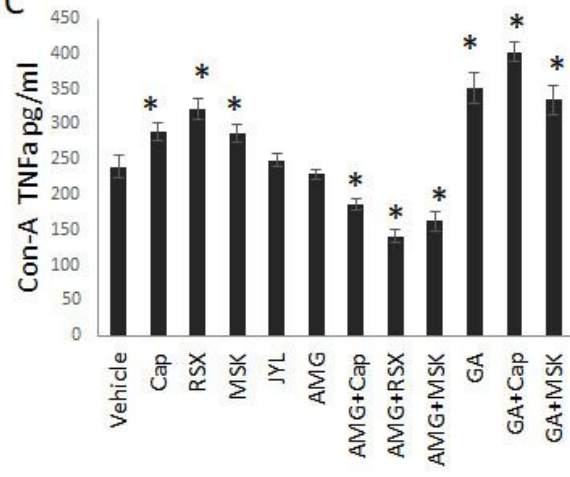

\section{Figure 1}

Effects of TRPV1 modulators and TrkA agonist on release of cytokines from mix leucocyte cultures (MLCs) of control mice. MLCs were prepared using spleen and lymph nodes of control mice and 
stimulated with ConA or LPS. MLCs were treated with Capsaicin (Cap) $(1 \mu \mathrm{M})$, resiniferatoxin (RSX)

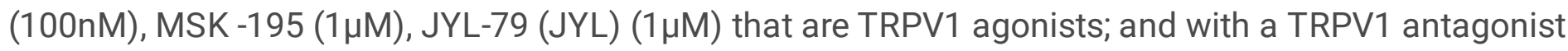

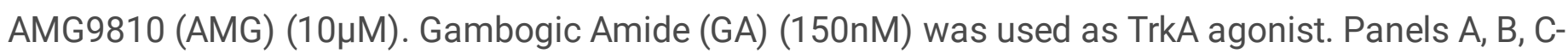
changes in secretion of IFN- $\gamma$, IL-17 and TNF-a following ConA challenge respectively. Panels D, Echanges in IL-10 and IL-6 secretion following LPS challenge. Treated groups were compared with the control (vehicle) group using ANOVA followed by Dunnett Multiple Comparisons test. ${ }^{*}<<0,05$ compared to vehicle.

A

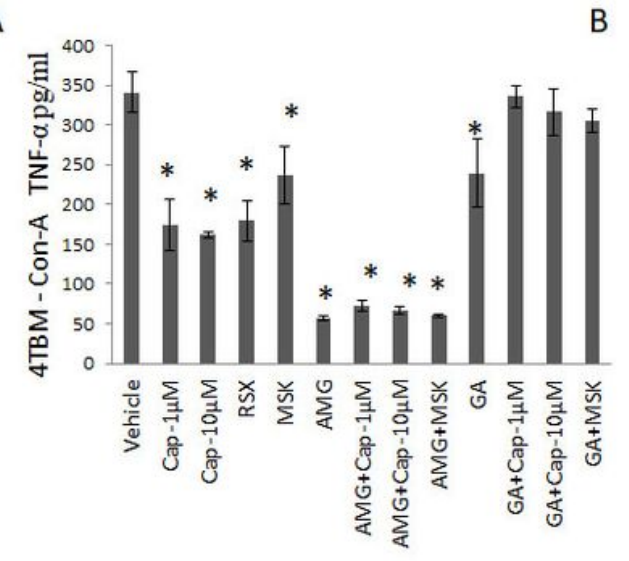

B

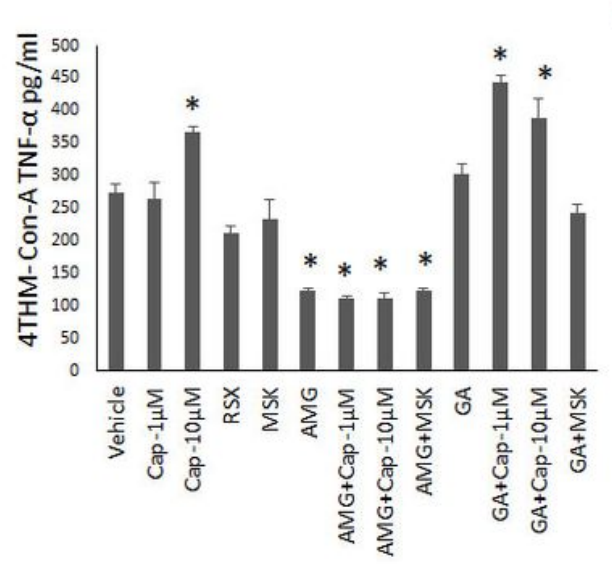

C

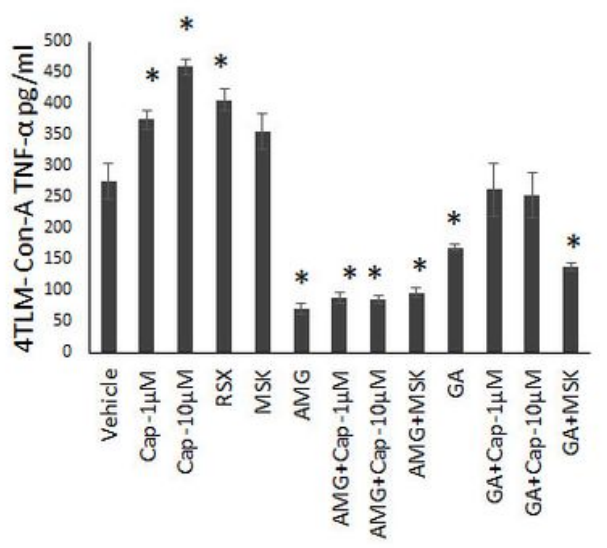

Figure 2

Effects of TRPV1 modulators and TrkA agonist on TNF-a release from mix leucocyte cultures (MLCs) of tumor-bearing mice. MLCs were prepared using spleen and lymph nodes of mice injected with 4TBM (Panel A), 4THM (Panel B) and 4TLM (Panel C) cells and stimulated with ConA. MLCs were treated with Capsaicin (Cap) $(1 \mu \mathrm{M}, 10 \mu \mathrm{M})$, resinferatoxin (RSX) (100nM), MSK-195 $(1 \mu \mathrm{M})$, that are TRPV1 agonists;

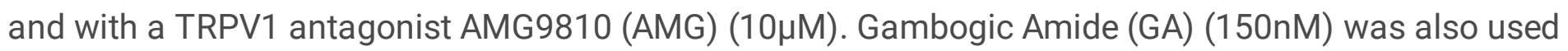
as a TrkA agonist. Changes in TNF-a secretion are seen following ConA challenge. Treated groups were compared with the control (vehicle) group using ANOVA followed by Dunnett Multiple Comparisons test. ${ }^{*} \mathrm{p}<0,05$ compared to vehicle.

A

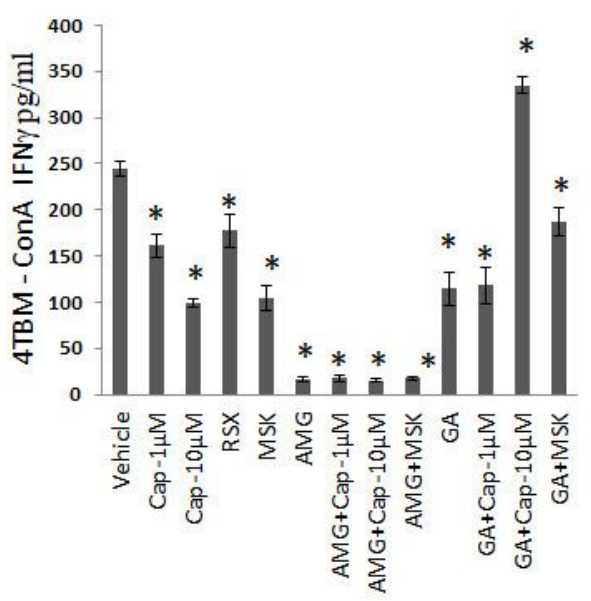

B

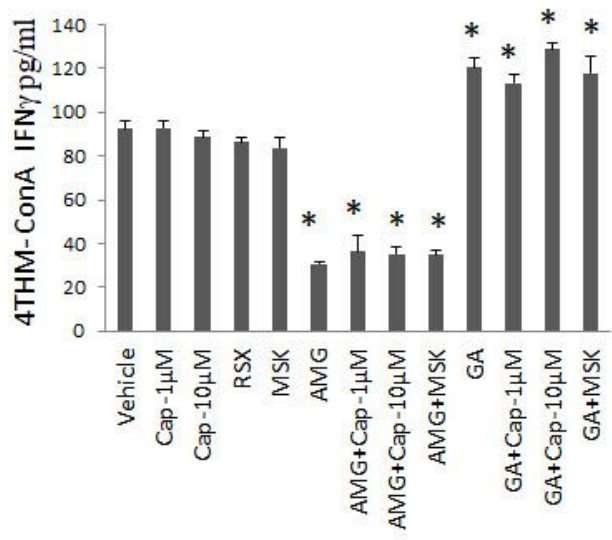

C

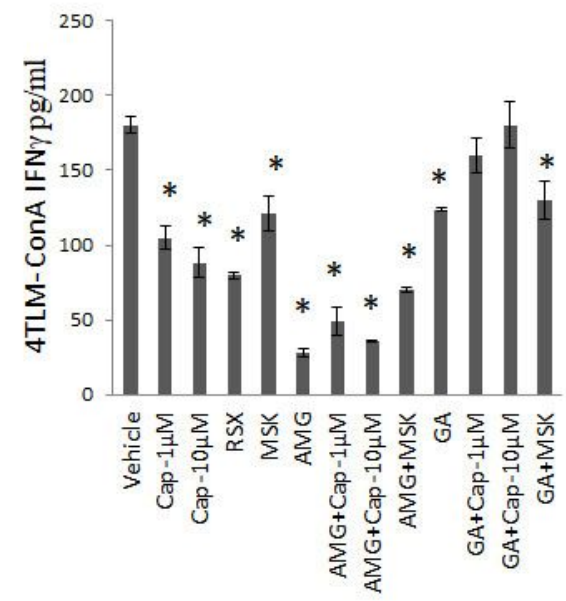




\section{Figure 3}

Effects of TRPV1 modulators and TrkA agonist on IFN-y release from mix leucocyte cultures (MLCs) of tumor-bearing mice. MLCs were prepared using spleen and lymph nodes of mice injected with 4TBM (Panel A), 4THM (Panel B) and 4TLM (Panel C) cells and stimulated with ConA. MLCs were treated with Capsaicin (Cap) $(1 \mu \mathrm{M}, 10 \mu \mathrm{M})$, resiniferatoxin (RSX) (100nM), MSK-195 $(1 \mu \mathrm{M})$, that are TRPV1 agonists;

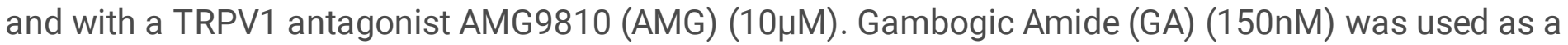
TrkA agonist. Changes in IFN-y secretion are seen following ConA challenge. Treated groups were compared with the control (vehicle) group using ANOVA followed by Dunnett Multiple Comparisons test. ${ }^{*} \mathrm{p}<0,05$ compared to vehicle.

A

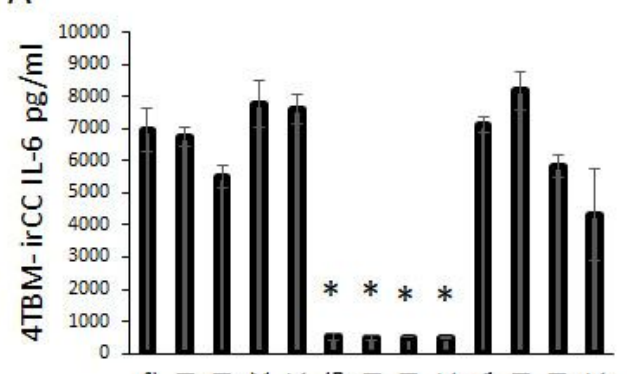

$\frac{\omega}{0} \sum \sum \times$ 药
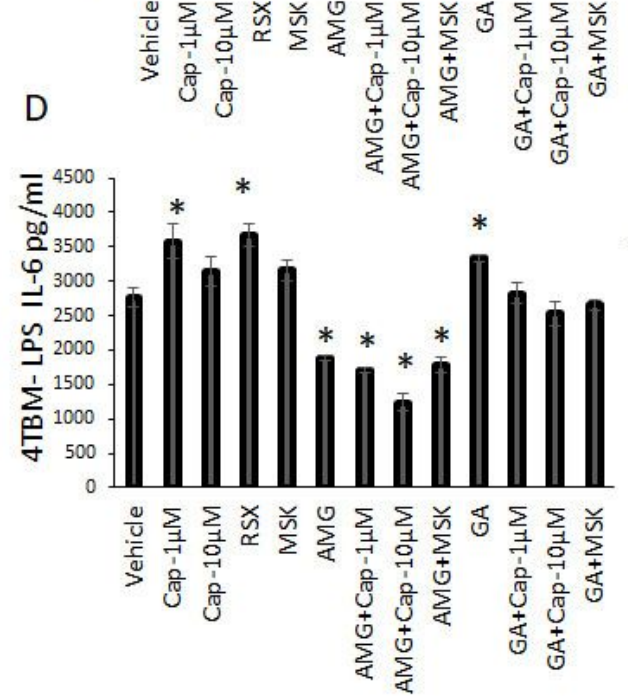

B
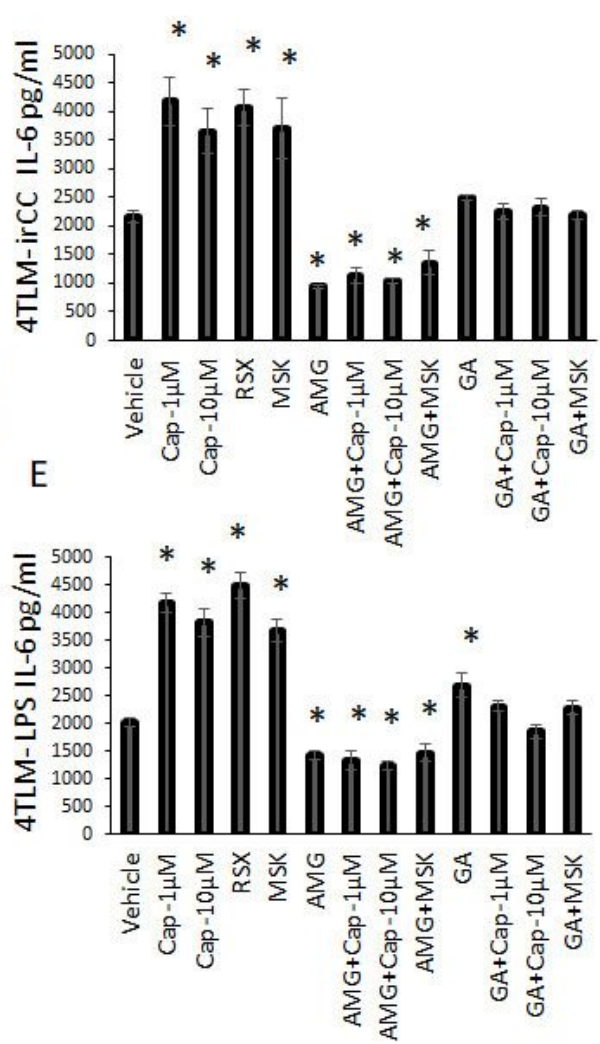

C
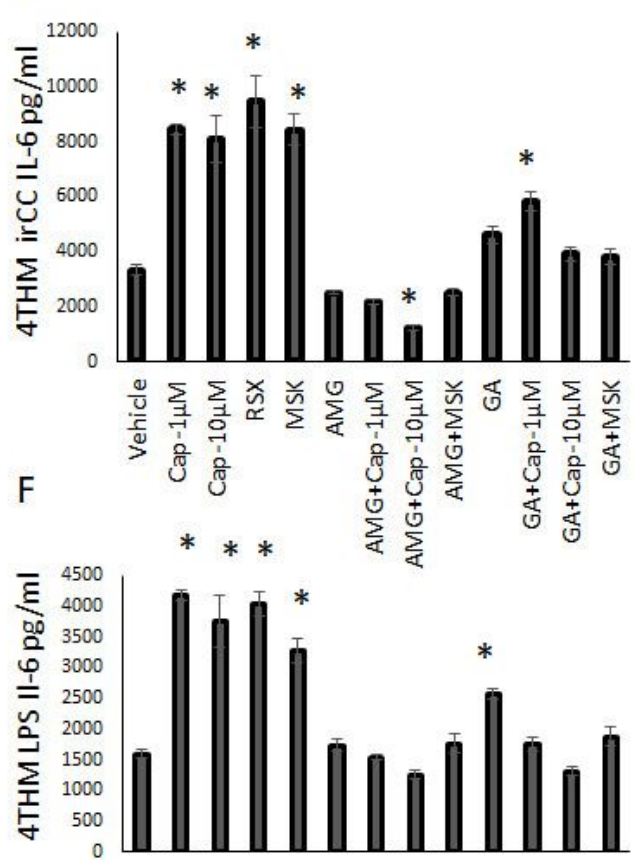

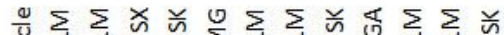

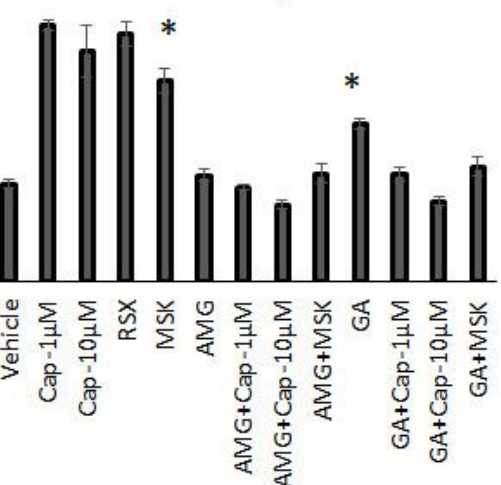

Figure 4

Effects of TRPV1 modulators and TrkA agonist on IL-6 release from mix leucocyte cultures (MLCs) of tumor-bearing mice. MLCs were prepared using spleen and lymph nodes of mice injected with 4TBM (Panel A, D), 4THM (Panel B, E) and 4TLM (Panel C, F) cells and stimulated with irradiated corresponding cancer cells (irCC) (Panels A-C) or with LPS (Panels D-E). MLCs were treated with Capsaicin (Cap) $(1 \mu \mathrm{M}$, $10 \mu \mathrm{M})$, resiniferatoxin (RSX) $(100 \mathrm{nM}), \mathrm{MSK}-195(1 \mu \mathrm{M})$, that are TRPV1 agonists; and with a TRPV1 antagonist AMG9810 (AMG) (10 $\mu$ M). Gambogic Amide (GA) (150nM) was used as a TrkA agonist. Changes in IL-6 secretion are seen following irCC (Panels A-C) and LPS (Panels D-F) challenge. Treated groups were compared with the control (vehicle) group using ANOVA followed by Dunnett Multiple Comparisons test. ${ }^{*} \mathrm{p}<0,05$ compared to vehicle. 
A
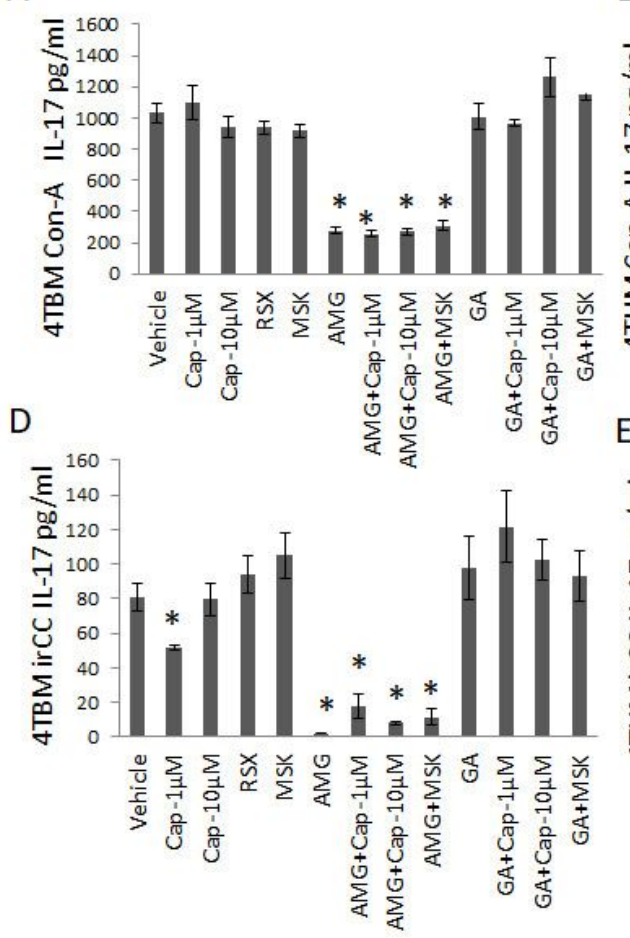

B

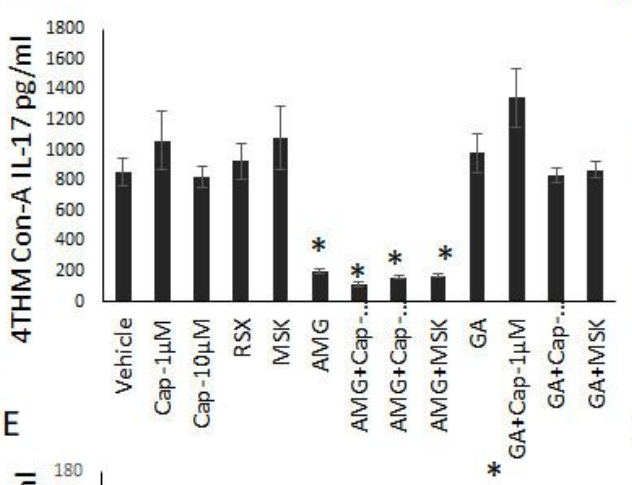

C
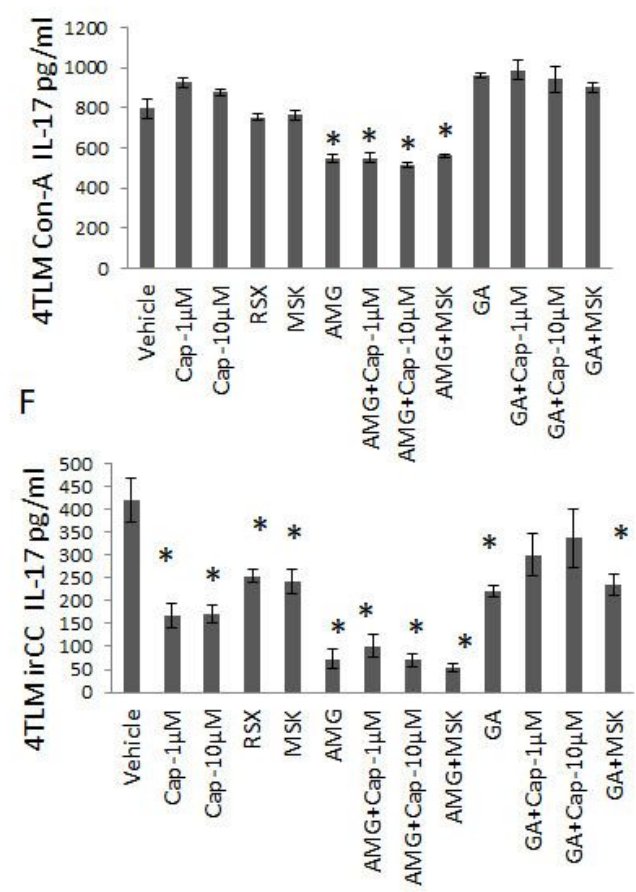

\section{Figure 5}

Effects of TRPV1 modulators and TrkA agonist on IL-17 release from mix leucocyte cultures (MLCs) of tumor-bearing mice. MLCs were prepared using spleen and lymph nodes of mice injected with 4TBM (Panel A, D), 4THM (Panel B, E) and 4TLM (Panel C, F) cells and stimulated with LPS (Panels A-C) or with irradiated corresponding cancer cells (irCC) (Panels D-E). MLCs were treated with Capsaicin (Cap) $(1 \mu \mathrm{M}$, $10 \mu \mathrm{M})$, resiniferatoxin (RSX) $(100 \mathrm{nM}), \mathrm{MSK}-195(1 \mu \mathrm{M})$, that are TRPV1 agonists; and with a TRPV1

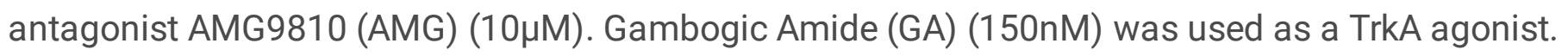
Changes in IL-17 secretion are seen following LPS (Panels A-C) and irCC (Panels D-F) challenge. Treated groups were compared with the control (vehicle) group using ANOVA followed by Dunnett Multiple Comparisons test. ${ }^{*} p<0,05$ compared to vehicle.

\section{Supplementary Files}

This is a list of supplementary files associated with this preprint. Click to download.

- Suppfiguresfinal.pdf 